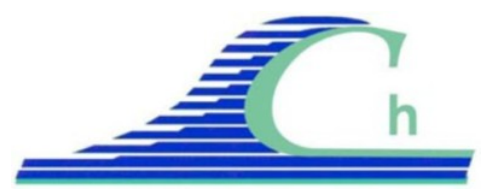

XII ${ }^{\text {èmes }}$ Journées Nationales Génie Côtier - Génie Civil

Cherbourg, 12-14 juin 2012

DOI:10.5150/jngcgc.2012.119-S @ Editions Paralia CFL

disponible en ligne - http://www.paralia.fr - available online

\title{
Evaluation environnementale de la réutilisation de sédiments de dragage en tant que matériau alternatif
}

\author{
Lucile SAUSSAYE $^{1,2}$, Hakim HAMDOUN ${ }^{2}$, Mohamed BOUTOUIL ${ }^{1,2}$, \\ Fabienne BARAUD ${ }^{2}$, Lydia LELEYTER ${ }^{2}$, \\ Walid MAHERZI ${ }^{1,2}$, Pierre-André LE GOFF ${ }^{3}$
}

1. ESITC Caen, 14610 Epron, France. ecr.geotech@esitc-caen.fr ; dir.recherche@esitc-caen.fr

2. Université de Caen Basse-Normandie, Unité de Recherche Aliments Bioprocédés Toxicologie Environnements (UR ABTE) EA 4651, 14032 Caen, France.

3. Laboratoire d'Analyses de Surveillance et d'Expertise de la Marine, 50100 Cherbourg Octeville, France.

\section{Résumé :}

Les résultats présentés dans cet article s’inscrivent dans le cadre du projet de recherche européen SETARMS dont l'un des objectifs est de mettre en place des solutions innovantes et durables de gestion des sédiments marins dragués en Manche (FranceAngleterre) pour une application en techniques routières.

L’utilisation de ces matériaux particuliers, assimilés à des déchets lors de leur extraction du milieu marin, requiert désormais une évaluation environnementale avant leur utilisation dans le domaine routier. En effet, le niveau de dangerosité du matériau peut limiter ses possibilités de réutilisation : un déchet qui serait dangereux ne peut trouver sa place dans un profil routier potentiellement drainé par les eaux d'infiltration. Les 8 sédiments français étudiés sont considérés comme non dangereux selon les critères du guide d'acceptabilité édité par le SETRA en 2011. Cette acceptabilité environnementale des sédiments ouvre ensuite sur des études géotechniques et environnementales de stabilisation/solidification à base de liants hydrauliques en laboratoire.

Un second niveau de validation est ensuite requis. En effet, si les performances géotechniques du matériau traité permettent de déclarer son aptitude à une utilisation en techniques routières, la valorisation ne peut être effective que s’il est démontré que le matériau alternatif ainsi formulé est écologiquement viable. L’approche est ici considérée malgré les faibles performances mécaniques enregistrées après un simple traitement aux liants hydrauliques, résultant d'une application directe des guides techniques de terrassements.

Mots-clés: Sédiments de dragage marins - Lixiviation - Acceptabilité environnementale - Valorisation - Techniques routières 


\section{Introduction}

Dans le cadre du projet SETARMS, la méthodologie d'étude mise en place doit permettre d'évaluer le potentiel de valorisation d'un sédiment de dragage marin en technique routière. Les partenaires du projet (lancé en octobre 2009), se sont organisés pour réaliser le prélèvement de treize sédiments, de part et d'autre de la Manche. Huit sédiments ont fait l'objet d'une caractérisation géotechnique, au sein des laboratoires de l'ESITC Caen et de l'Ecole des Mines de Douai, et d'une caractérisation physicochimique au sein du laboratoire Aliments Bioprocédés Toxicologie Environnements ABTE de l'Université de Caen Basse-Normandie (UCBN) et du Laboratoire d'Analyses, de Surveillance et d'Expertise de la Marine (LASEM) de Cherbourg. L'étude s'est poursuivie en 2011 par un suivi géotechnique de l'aptitude au traitement aux liants hydrauliques. L’étude physico-chimique des sédiments traités est réalisée à l'ABTE.

Depuis mars 2011, le SETRA (Service d'Etudes sur les Transports, les Routes et leurs Aménagements) appuie le potentiel de valorisation de matériaux alternatifs en techniques routières par la définition de leur acceptabilité environnementale. Il rappelle ainsi que les sédiments de dragage marins sont des déchets dès lors qu’ils sont émergés. Leur niveau de dangerosité doit être évalué selon les seuils de la Décision du Conseil 2003/33/CE du 19/12/2002 établissant des critères et des procédures d'admission des déchets en décharge conformément à l'article 16 et à l'annexe II de la directive 1999/31/CE, parue au JOCE du 16 janvier 2003. Le guide d'acceptabilité des matériaux alternatifs en technique routière (SETRA, 2011) vise alors "à ne pas faire de la route un substitut de mise en décharge". Les premiers niveaux d'évaluation environnementale intègrent une analyse de la fraction organique totale et de la fraction soluble à l'eau des matériaux bruts et des matériaux alternatifs. Avant la parution du guide, les travaux de recherche visant à démontrer le potentiel de valorisation des sédiments de dragage s'intéressaient déjà à la fraction soluble à l'eau, avant traitement et après traitement (BRAKNI, 2008 ; NGUYEN, 2008).

Les résultats présentés dans cet article sont extraits des caractérisations physicochimiques réalisées sur huit sédiments bruts et traités aux liants hydrauliques, après 180 jours de cure. La fraction organique des sédiments bruts a été analysée conjointement par l'ABTE (carbone organique total COT) et le LASEM (polychlorobiphényles (PCB, hydrocarbure aromatiques polycycliques (HAP) et hydrocarbures totaux (HCT)). Les fractions solubles à l'eau ont été extraites et analysées à l'ABTE. Les données sont ici comparées aux seuils des niveaux 1 et 2 du guide SETRA.

\section{Matériaux et méthodes}

\subsection{Composés organiques totaux}

Le niveau 1 fait appel à la détermination des teneurs en composés organiques totaux. La détermination du COT est réalisée selon la norme ISO 14235 modifiée. La méthode se 


\section{XII ${ }^{\text {èmes }}$ Journées Nationales Génie Côtier - Génie Civil \\ Cherbourg, 12-14 juin 2012}

base sur une oxydation du COT par le dichromate de potassium $\left(\mathrm{K}_{2} \mathrm{Cr}_{2} \mathrm{O}_{7}\right)$ en milieu acide puis par un dosage par spectrophotométrie (Pharmacia Biotech Novaspec II).

La détermination des teneurs en PCB est réalisée selon la norme XP X 33-012. Les PCB sont extraits dans un mélange acétone/hexane et analysés par chromatographie en phase gazeuse couplée à un spectromètre de masse (CPG/SM).

Le dosage des HAP est réalisé selon la norme XP X 33-012. Après extraction dans un mélange acétone/hexane, les échantillons sont analysés par chromatographie liquide haute performance (HPLC). La teneur en HCT est mesurée selon un mode opératoire interne au LASEM. Après extraction dans du perchloroéthylène $\left(\mathrm{C}_{2} \mathrm{Cl}_{4}\right)$, les HCT sont dosés par spectroscopie Infrarouge à Transformée de Fourier (IRTF).

\subsection{Extractions simples à l'eau ultra pure}

Le niveau 1 fait aussi appel à la détermination de concentrations en métaux et en anions de la fraction soluble à l'eau des sédiments bruts et traités. Afin d'extraire cette fraction, une lixiviation à l'eau ultra pure (EUP) est réalisée selon la norme NF EN 12457-2. La fraction 0/4 mm d'échantillons de matériau broyé est mise au contact d'EUP avec un ratio liquide/solide $(\mathrm{L} / \mathrm{S})$ de 10 . L'extraction est réalisée sur table rotative, à $200 \operatorname{tr~min}^{-1}$, pendant 24 heures. Les lixiviats sont filtrés à $0,45 \mu \mathrm{m}$ puis conservés à 4 ${ }^{\circ} \mathrm{C}$ avant analyse. Le niveau 2 appelle l'utilisation d'un second protocole pour les sédiments traités: la fraction soluble à l'eau est extraite sur monolithe (éprouvette cylindrique de diamètre $5 \mathrm{~cm}$ et de hauteur $5 \mathrm{~cm}$ ) avec un ratio $\mathrm{L} / \mathrm{S}$ de 10 , selon la norme XP X 31-211. Les concentrations élémentaires en As, Ba, Cd, Cr, Cu, Mo, Ni, Pb et $\mathrm{Zn}$ de la fraction soluble à l'eau sont analysées par spectrométrie d'émission atomique par induction plasma (ICP-AES, Varian, Vista MPX). Les concentrations anioniques en $\mathrm{F}^{-}$, en $\mathrm{Cl}^{-}$et en $\mathrm{SO}_{4}{ }^{2-}$ sont analysées par chromatographie ionique (Dionex). En raison des fortes teneurs en $\mathrm{Cl}^{-}$des sédiments marins, les échantillons sont préalablement dilués au centième. Lors de l'écriture de cet article, les concentrations anioniques n’ont pas été mesurées sur monolithe.

\section{Résultats}

\subsection{Paramètres physico-chimiques des sédiments bruts}

Le tableau 1 donne les concentrations élémentaires et anioniques de la fraction soluble à l'eau des huit sédiments étudiés ainsi que leurs teneurs en composés organiques totaux. La colonne grise indique les valeurs de concentrations seuils proposées par le guide pour valider une réutilisation de matériaux en techniques routières. Pour l'ensemble des sédiments, les concentrations en éléments solubles sont inférieures aux seuils proposés par le guide pour une réutilisation en techniques routières. A noter que les concentrations en $\mathrm{As}, \mathrm{Cd}, \mathrm{Cr}, \mathrm{Pb}$ et $\mathrm{Zn}$ sont inférieures aux limites de détections (LD). 
Pour des matériaux naturellement riches en chlorures et en sulfates, ce qui est le cas de sédiments de dragage marins, le guide SETRA propose d'évaluer l'acceptabilité environnementale par comparaison à la somme des concentrations en chlorures et en sulfates, regroupées sous l'appellation "fraction soluble". La seule prise en compte de la concentration en ions $\mathrm{Cl}^{-}$obligerait à considérer les sédiments B001, C001, D001, G001 et M001 comme des déchets dangereux. La fraction soluble permet en revanche de classer l'ensemble des sédiments comme des déchets non dangereux et de valider ainsi leur réutilisation en techniques routières. Par ailleurs, l'impact environnemental des chlorures peut être minimisé dans le cas où la réalisation d'un remblai portuaire serait envisagée (AGOSTINI, 2006).

Par ailleurs, les teneurs en COT, en PCB et en HAP des sédiments bruts sont inférieures au seuil d'acceptabilité. Cependant, pour l'ensemble des sédiments, les teneurs en HCT franchissent le seuil de $500 \mathrm{mg} \mathrm{kg}^{-1}$ de matière sèche.

Tableau 1. Teneurs moyennes en éléments traces et en anions de la fraction soluble à l'eau et teneurs en composés organiques totaux des sédiments bruts (exprimées en mg kg-1 de matière sèche).

\begin{tabular}{|c|c|c|c|c|c|c|c|c|c|c|}
\hline & $m g \mathrm{~kg}^{-1}$ & ${ }^{1} L D$ & A001 & B001 & C001 & D001 & E001 & F001 & G001 & M001 \\
\hline As & 2 & 0.22 & $<L D$ & $<L D$ & $<L D$ & $<L D$ & $<L D$ & $<L D$ & $<L D$ & $<L D$ \\
\hline$B a$ & 100 & 0.03 & 0.2 & 0.3 & 0.2 & 0.1 & 0.2 & 0.2 & 0.3 & 0.2 \\
\hline$C d$ & 1 & 0.004 & $<L D$ & $<L D$ & $<L D$ & $<L D$ & $<L D$ & $<L D$ & $<L D$ & $<L D$ \\
\hline $\mathrm{Cr}$ & 10 & 0.02 & $<L D$ & $<L D$ & $<L D$ & $<L D$ & $<L D$ & $<L D$ & $<L D$ & $<L D$ \\
\hline $\mathrm{Cu}$ & 50 & 0.02 & 0.2 & 0.1 & 0.2 & 0.3 & 0.2 & 0.1 & 0.1 & 0.1 \\
\hline Mo & 10 & 0.06 & 1.1 & 1.2 & 1.1 & 1.3 & 1.4 & 1.2 & 1.0 & 1.2 \\
\hline $\mathrm{Ni}$ & 10 & 0.08 & 0.2 & $<L D$ & 0.1 & 0.1 & 0.1 & 0.1 & 0.1 & 0.1 \\
\hline$P b$ & 10 & 0.09 & $<L D$ & $<L D$ & $<L D$ & $<L D$ & $<L D$ & $<L D$ & $<L D$ & $<L D$ \\
\hline Zn & 50 & 0.01 & $<L D$ & $<L D$ & $<L D$ & $<L D$ & $<L D$ & $<L D$ & $<L D$ & $<L D$ \\
\hline Fluorure & 150 & I & $N D$ & $N D$ & $N D$ & $N D$ & $N D$ & $N D$ & $N D$ & $N D$ \\
\hline Chlorure & 15000 & I & 10901 & 33440 & 19283 & 20447 & 6570 & 7739 & 32776 & 31846 \\
\hline Sulfate & 20000 & I & 133 & 2516 & 1871 & 1993 & 118 & 197 & 2710 & 2530 \\
\hline Fract. soluble & 60000 & / & 11034 & 35956 & 21154 & 22440 & 6688 & 7936 & 35487 & 34376 \\
\hline COT & 60000 & / & 21000 & 38000 & 58000 & 49000 & 60000 & 37000 & 47000 & 30000 \\
\hline PCB & 1 & 0,007 & 0.009 & 0.125 & 0.071 & 0.460 & 0.166 & 0.101 & 0.032 & 0.057 \\
\hline HAP & 50 & 0,030 & 3.4 & 11.7 & 31.6 & 4.3 & 5.1 & 3.3 & 10.1 & 2.7 \\
\hline HCT & 500 & 100 & 581 & 613 & 2190 & 841 & 1234 & 1438 & 572 & 2135 \\
\hline \multicolumn{11}{|c|}{ matériaux inertes ou non dangereux : validation d'une réutilisation en technique routière } \\
\hline & \multicolumn{10}{|c|}{ matériaux dangereux : rejet d'une réutilisation en technique routière } \\
\hline
\end{tabular}

A la lecture du guide, il apparaît que les modes opératoires utilisés ne correspondent pas à ceux des normes indiquées par le guide pour les PCB, HAP et HCT (NF EN 15308, 


\section{XII ${ }^{\text {èmes }}$ Journées Nationales Génie Côtier - Génie Civil \\ Cherbourg, 12-14 juin 2012}

NF EN 14039 et NF EN 15527 respectivement). Ainsi, les HCT mesurés par le LASEM englobent une plus large gamme d'hydrocarbures que les seuls C10 à C40 mesurés par l'application de la norme NF EN 15527. Le dépassement du seuil peut être lié à cette différence de protocole. Il n'est donc pas possible de conclure quant à la dangerosité des sédiments bruts, y compris pour les sédiments C001, E001, F001 et M001 pour lesquels les teneurs semblent bien plus importantes que le seuil de $500 \mathrm{mg} \mathrm{kg}^{-1}$.

\subsection{Caractérisation des sédiments traités}

3.2.1 Aptitude géotechnique à la réutilisation des sédiments de dragage en couche de forme

Les paramètres géotechniques d'une réutilisation de matériaux en couche de forme sont transcrits dans le Guide technique des Terrassements Routiers (GTR) (LCPC-SETRA, 1992) et dans le Guide technique de Traitement des Sols - application aux remblais et couche de forme (GTS) (LCPC-SETRA, 2000). La caractérisation géotechnique des sédiments a été abordée par MAHERZI et al. (2011). Les huit sédiments français sont considérés comme des sous-produits industriels faiblement à fortement organiques de classe F11 à F12. Leur comportement géotechnique est assimilé à celui de sols fins de classe A1 ou A2, avec une réactivité de la fraction argileuse relativement modérée.

La définition d'une formulation de traitement avec $3 \%$ de chaux vive et $6 \%$ de ciment CEMII 32,5 par rapport à la masse de sédiment sec a été abordée par BOUTOUIL et al. (2011). Aucun des sédiments soumis à ce simple traitement aux liants hydrauliques ne peut être considéré apte à une réutilisation en couche de forme. Précisons que le guide d'acceptabilité n'admet pas la dilution et/ou la stabilisation par l'apport de correcteur et/ou de liants hydrauliques comme une méthode de traitement valide si elle s'applique à un déchet dangereux. Les tests de lixiviation à l'eau réalisés sur les sédiments traités vont permettre de se positionner par rapport aux seuils des niveaux 1 et 2 .

\subsubsection{Aptitude environnementale à la réutilisation des sédiments de dragage en couche} de forme

Le tableau 2 reporte les concentrations en éléments traces et en ions $\mathrm{F}^{-}, \mathrm{Cl}^{-}$et $\mathrm{SO}_{4}{ }^{2-}$ de la fraction soluble à l'eau des sédiments traités. Il met en parallèle les résultats de teneurs en éléments traces des lixiviats obtenues sur poudre et sur monolithe. Selon le guide d'acceptabilité, le premier type d'essai permet de franchir l'étape de caractérisation de niveau 1, pour une réutilisation du matériau en techniques routières. Le second, de type lixiviation surfacique, permet de valider ou non l'étape de niveau 2, pour une réutilisation du matériau en couche de forme.

Les 8 sédiments traités présentent des concentrations en éléments traces solubles à l'eau inférieures aux seuils des niveaux 1 et 2. Les concentrations en $\mathrm{Cd}, \mathrm{Cr}, \mathrm{Pb}$ et $\mathrm{Zn}$ sont à nouveau en dessous des LD. Les concentrations en ions $\mathrm{Cl}^{-}$sont inférieures au seuil du 
niveau 1 pour les sédiments C001, F001 et G001. Elles sont supérieures au seuil pour les sédiments A001, B001, D001, E001 et M001. Les teneurs en anions de la fraction soluble restent cependant inférieures au seuil de niveau 1.

Les concentrations en $\mathrm{Cl}^{-}$relargués diminuent pour les sédiments B001, C001, D001, G001 et M001 par rapport aux sédiments non traités. A l'exception du sédiment E001, les sulfates lessivés ne sont pas détectés après traitement. La LD de l'anion étant très basse, le facteur de dilution des lixiviats ne peut pas être invoqué. Cette évolution pourrait signifier une rétention des sulfates par les phases cimentaires. Les fluorures, précédemment absents de la fraction soluble à l'eau des sédiments bruts, ne sont pas détectés non plus après traitement.

Tableau 2. Teneurs moyennes en éléments traces et en anions de la fraction solubles à l'eau des sédiments traités aux liants hydrauliques (en $\mathrm{mg} \mathrm{kg}^{-1}$ de matière sèche) - par lixiviation sur poudre / par lixiviation surfacique.

\begin{tabular}{|c|c|c|c|c|c|c|c|c|c|c|}
\hline & $m g \cdot \mathrm{kg}^{-1}$ & $L D$ & $A 001$ & B001 & $C 001$ & D001 & E001 & F001 & G001 & M001 \\
\hline As & $2 / 0.8$ & 0.22 & $\begin{array}{l}0.2 / \\
0.1\end{array}$ & $\begin{array}{l}0.4 / \\
0.3\end{array}$ & $\begin{array}{l}<L D / \\
0.2\end{array}$ & $\begin{array}{l}0.4 / \\
0.1\end{array}$ & $\begin{array}{l}0.2 / \\
0.5\end{array}$ & $\begin{array}{l}<L D / \\
0.1\end{array}$ & $\begin{array}{l}<L D / \\
0.1\end{array}$ & $\begin{array}{l}<L D / \\
0.2\end{array}$ \\
\hline$B a$ & $100 / 56$ & 0.03 & $\begin{array}{l}0.2 / \\
0.2\end{array}$ & $\begin{array}{l}0.4 / \\
0.4\end{array}$ & $\begin{array}{l}0.1 / \\
0.2\end{array}$ & $\begin{array}{l}0.2 / \\
0.6\end{array}$ & $\begin{array}{l}0.6 / \\
0.3\end{array}$ & $\begin{array}{l}0.2 / \\
0.2\end{array}$ & $\begin{array}{l}0.2 / \\
0.3\end{array}$ & $0.2 / 0.2$ \\
\hline$C d$ & $1 / 0.32$ & 0.004 & $\begin{array}{l}<L D / \\
<L D\end{array}$ & $\begin{array}{l}<L D / \\
<L D\end{array}$ & $\begin{array}{l}<L D / \\
<L D\end{array}$ & $\begin{array}{l}<L D / \\
<L D\end{array}$ & $\begin{array}{l}<L D / \\
<L D\end{array}$ & $\begin{array}{l}<L D / \\
<L D\end{array}$ & $\begin{array}{l}<L D / \\
<L D\end{array}$ & $\begin{array}{l}<L D / \\
<L D\end{array}$ \\
\hline $\mathrm{Cr}$ & $10 / 4$ & 0.02 & $\begin{array}{l}<L D / \\
<L D\end{array}$ & $\begin{array}{l}<L D / \\
<L D\end{array}$ & $\begin{array}{l}<L D / \\
<L D\end{array}$ & $\begin{array}{l}<L D / \\
<L D\end{array}$ & $\begin{array}{l}<L D / \\
<L D\end{array}$ & $\begin{array}{l}<L D / \\
<L D\end{array}$ & $\begin{array}{l}<L D / \\
<L D\end{array}$ & $\begin{array}{l}<L D / \\
<L D\end{array}$ \\
\hline $\mathrm{Cu}$ & $50 / 50$ & 0.02 & $\begin{array}{l}1.0 / \\
1.0\end{array}$ & $\begin{array}{l}1.7 / \\
1.6 \\
\end{array}$ & $\begin{array}{l}0.4 / \\
0.8\end{array}$ & $\begin{array}{l}0.7 / \\
0.7\end{array}$ & $\begin{array}{l}0.7 / \\
0.2\end{array}$ & $\begin{array}{l}0.7 / \\
1.0\end{array}$ & $\begin{array}{l}0.7 / \\
1.1\end{array}$ & $0.8 / 1.1$ \\
\hline Mo & $10 / 5.6$ & 0.06 & $\begin{array}{l}1.5 / \\
1.6\end{array}$ & $\begin{array}{l}1.8 / \\
1.8\end{array}$ & $\begin{array}{l}0.6 / \\
1.2\end{array}$ & $\begin{array}{l}1.8 / \\
1.3\end{array}$ & $\begin{array}{l}1.3 / \\
1.5\end{array}$ & $\begin{array}{l}1.2 / \\
1.5\end{array}$ & $\begin{array}{l}1.1 / \\
1.7\end{array}$ & $1.2 / 1.7$ \\
\hline$N i$ & $10 / 1.6$ & 0.08 & $\begin{array}{l}0.5 / \\
0.6\end{array}$ & $\begin{array}{l}0.8 / \\
0.8\end{array}$ & $\begin{array}{l}0.1 / \\
0.4\end{array}$ & $\begin{array}{l}0.8 / \\
0.3\end{array}$ & $\begin{array}{l}0.3 / \\
<L D\end{array}$ & $\begin{array}{l}0.4 / \\
0.5\end{array}$ & $\begin{array}{l}0.3 / \\
0.6\end{array}$ & $0.4 / 0.6$ \\
\hline$P b$ & $10 / 0.8$ & 0.09 & $\begin{array}{l}<L D / \\
<L D\end{array}$ & $\begin{array}{l}<L D / \\
<L D\end{array}$ & $\begin{array}{l}<L D / \\
<L D\end{array}$ & $\begin{array}{l}<L D / \\
<L D\end{array}$ & $\begin{array}{l}<L D / \\
<L D\end{array}$ & $\begin{array}{l}<L D / \\
<L D\end{array}$ & $\begin{array}{l}<L D / \\
<L D\end{array}$ & $\begin{array}{l}<L D / \\
<L D\end{array}$ \\
\hline$Z n$ & $50 / 50$ & 0.01 & $\begin{array}{l}<L D / \\
<L D\end{array}$ & $\begin{array}{l}<L D / \\
<L D\end{array}$ & $\begin{array}{l}<L D / \\
<L D\end{array}$ & $\begin{array}{l}<L D / \\
<L D\end{array}$ & $\begin{array}{l}<L D / \\
<L D\end{array}$ & $\begin{array}{l}<L D / \\
<L D\end{array}$ & $\begin{array}{l}<L D / \\
<L D\end{array}$ & $\begin{array}{l}<L D / \\
<L D\end{array}$ \\
\hline Fluorure & 150 & 1 & $N D$ & $N D$ & $N D$ & $N D$ & $N D$ & $N D$ & $N D$ & $N D$ \\
\hline Chlorure & 15000 & 1 & 15802 & 19603 & 13794 & 19370 & 37739 & 11597 & 14900 & 16816 \\
\hline Sulfate & 20000 & 1 & $N D$ & $N D$ & $N D$ & $N D$ & 3037 & $N D$ & $N D$ & $N D$ \\
\hline Fract soluble & 60000 & 1 & 15802 & 19684 & 13794 & 19370 & 40776 & 11597 & 14900 & 16816 \\
\hline \multicolumn{11}{|c|}{ matériaux inertes ou non dangereux : validation d'une réutilisation en technique routière } \\
\hline
\end{tabular}




\section{XII ${ }^{\text {èmes }}$ Journées Nationales Génie Côtier - Génie Civil \\ Cherbourg, 12-14 juin 2012}

\section{Conclusions et perspectives}

Dans le cadre du projet SETARMS, la caractérisation géotechnique et physico-chimique des sédiments amène à considérer le comportement à la lixiviation de sédiments de dragage marins bruts ou traités aux liants hydrauliques.

Les premiers niveaux du guide d'acceptabilité pour l'utilisation des matériaux alternatifs en technique routière (SETRA, 2011) incitent à se préoccuper des caractéristiques physico-chimiques de la fraction soluble à l'eau du matériau à valoriser pour appréhender son impact environnemental.

La comparaison entre les analyses menées au cours des années 2010 et 2011 et les valeurs de concentrations des seuils proposés par le SETRA en 2011 donnent lieu aux conclusions suivantes :

- Les concentrations en éléments traces, en ions fluorures (concentrations nulles) et en anions $\left(\mathrm{Cl}^{-}+\mathrm{SO}_{4}{ }^{2-}\right)$ de la fraction soluble à l'eau des 8 sédiments bruts étudiés sont inférieures aux seuils de réutilisation en techniques routières ;

- Les teneurs en COT, en PCB et en HAP des 8 sédiments bruts sont acceptables ;

- La teneur en HCT est le seul critère qui tend à classer les sédiments comme "nondangereux" donc non inertes. Or, il est sans doute surévalué et de nouvelles analyses doivent donc être réalisées pour confirmer cette hypothèse ;

- Pour valider une réutilisation des sédiments bruts en techniques routières, des analyses complémentaires en Hg, Se, Sb et BTEX doivent par ailleurs être menées ;

- Les caractéristiques mécaniques des sédiments traités aux liants hydrauliques sont insuffisantes pour une réutilisation en couche de forme ;

- Les concentrations en éléments traces de la fraction soluble à l'eau des sédiments traités après 180 jours de cure normale sont inférieures aux seuils de niveaux 1 et 2 proposés par le guide : sur ce seul critère, les matériaux pourraient être acceptables pour une réutilisation en couche de forme ;

- Les concentrations en fluorures et en sulfates de la fraction soluble à l'eau sont faibles voire nulles après traitement ;

- Les concentrations en chlorures élevées sont compensées dans la fraction soluble, somme des concentrations en ions chlorures et sulfates ;

- Les concentrations anioniques des lixiviats sur monolithe n’ont pas été mesurées ne permettant pas de se positionner sur le niveau 2.

Ainsi, les analyses physico-chimiques, réalisées par l'UCBN et le LASEM, permettent a priori de se placer dans un scénario de réutilisation en techniques routières, voire de couche de forme, sous réserve d'un certain nombre d'analyses complémentaires, notamment en ce qui concerne la fraction organique totale. Le suivi environnemental des matériaux envisagé dans le cadre du projet se base sur des travaux antérieurs consacrés à la valorisation de déchets en techniques routières (FALLMAN \& AUREL, 1996 ; LEWIN, 1996), au travers de tests de lixiviation beaucoup plus agressifs $\left(\mathrm{HNO}_{3}\right.$ à $\mathrm{pH}$ 4). Toutefois aucun essai de lixiviation en laboratoire ne permettra de simuler les 
conditions réelles auxquelles sera soumis le matériau (VAN DER SLOOT et al., 1996). Au cours de l'année 2012, seront mises en œuvre des planches expérimentales afin d'assurer un suivi environnemental in situ.

\section{Remerciements}

Les auteurs remercient l'ensemble des partenaires du projet SETARMS ainsi que les organismes financeurs (Europe et Conseil Régional de Basse-Normandie).

\section{Références bibliographiques}

AGOSTINI F. (2006). Inertage et valorisation des sédiments de dragage marins. Thèse de doctorat, Université de Lille, 208 p.

BOUTOUIL M., BEN ABDELGHANI F., SAUSSAYE L., MAHERZI W. (2011). Étude du comportement de sédiments de dragage marins à l'essai d'aptitude au traitement. Conférence Méditerranéenne Côtière et Maritime, 22-24 novembre 2011, Tanger, Maroc, pp 141-144

BRAKNI S. (2008). Première approche vers une valorisation de granulats artificiels à base de sédiments de dragage, application en génie côtier. Thèse de doctorat, Ecole Nationale Supérieure des Mines de Douai, 182 p.

FALLMAN A.-M., AUREL B. (1996). Leaching test for environmental assessment of inorganic substances in wastes, Sweden. The Science of the Total Environment, Vol. 178, pp. 71-84

LCPC-SETRA (1992). Réalisation des remblais et des couches de forme - Guide technique (GTR). Fascicules I et II, 204 p.

LCPC-SETRA (2000). Traitement des sols à la chaux et/ou liants hydrauliques. Application à la réalisation des remblais et des couches de formes - Guide technique (GTS). 240 p.

LEWIN K. (1996). Leaching tests for waste compliance and characterisation: recent practical experiences. The Science of the Total Environment, Vol. 178, pp 85-89

SETRA (2011). Guide méthodologique acceptabilité de matériaux alternatifs en technique routière. $32 \mathrm{p}$.

MAHERZI W., BEN ABDELGHANI F., BOUTOUIL M., SAUSSAYE L. (2011). Sustainable Environmental Treatment And Reuse of Dredged Marine Sediments in Road Construction, ISWA 2011, 17-20 octobre 2011, Daegu, Corée, pp 456-466.

NGUYEN T. B. (2008). Valorisation des sédiments de dragage traités par le procédé dans des matériaux d'assises de chaussée - comportement mécanique et environnemental. Thèse de doctorat. Institut National des Sciences Appliquées de Toulouse, $244 \mathrm{p}$.

VAN DER SLOOT H.A., COMANS R.N.J., HJELMAR O. (1996). Similarities in the leaching behaviour of trace contaminants from waste, stabilized waste, construction materials and soils. The Science of the Total Environment, Vol. 178, pp 111-126 